\title{
Cyclostationary Feature Detection in Cognitive Radio using Different Modulation Schemes
}

\author{
Aparna P.S \\ II ME-Communication Systems \\ ECE Department \\ SNS College of Technology \\ Vazhiyampalayam \\ Coimbatore-641035
}

\author{
M. Jayasheela \\ Associate Professor \\ ECE Department \\ SNS College of Technology \\ Vazhiyampalayam \\ Coimbatore-641035
}

\begin{abstract}
The various spectrum sensing schemes involved in cognitive radio have always been researched and discussed. An ideal detection scheme should be fast, accurate and efficient. Cyclostationary feature detection is a detection scheme that satisfies all these criteria. The method also possesses the ability to distinguish between noise and the primary user signal. One major advantage of cyclostationary feature detection method is that in addition to identifying the primary user signal, it also identifies the modulation scheme used by the primary user. This paper investigates the cyclostationary feature detection method under different modulation schemes. In this paper, cooperative spectrum sensing is performed which involves the cooperation among various cognitive relay nodes. Cooperative spectrum sensing is thus found to be an effective technique to improve the detection performance by exploring the spatial diversity of various relay nodes.
\end{abstract}

\section{General Terms}

Cognitive radio spectrum sensing

\section{Keywords}

Cognitive radio, Spectrum sensing, Cyclostationary Feature Detection, Cyclic Spectral Correlation Function, Cooperative spectrum sensing, Primary User.

\section{INTRODUCTION}

The cognitive radio concept dates back to 1998 when the idea was first conceived by Sir Joseph Mitola III at the Royal Institute Of Technology in Stockholm. The major driving factor behind was the ever increasing requirement for the radio spectrum. There was an unusual drive for improved communication speeds. The only way to provide communication flexibility was by efficiently utilizing the radio spectrum.

Cognitive Radio turns out to be a solution to the spectral crowding problem by introducing the opportunistic usage of frequency bands. These frequency bands must not be occupied by licensed users. One most important component of a cognitive radio is its ability to measure, sense, learn, and be aware of the parameters related to the radio channel characteristics, the availability of radio spectrum as well as power, the user requirements and their applications, and also other operating restrictions. In cognitive radio terminology, primary user (PU) can be defined as the user who possesses higher priority on the usage of any specific part of the spectrum. Secondary user is the one with much lower priority. The secondary user gets access to this spectrum in such a way that it do not cause any sort of interference to the already existing primary user. Therefore a secondary user should have cognitive radio capabilities, such as sensing the spectrum reliably so as to check whether the band of the spectrum is being used by any primary user and to change its own radio parameters in order to exploit the unused band of the spectrum.

Spectrum sensing can be described as an art of performing measurements on a part of the radio spectrum and forming a decision related to the spectrum usage based upon the earlier measured data. Now a day, the service providers face a situation in which they require a larger amount of spectrum to satisfy the increasing quality of service (QoS) requirements of the users. This is the reason for the increased interest in unlicensed spectrum access, and spectrum sensing is an important enabler for this. Spectrum sensing is to detect the presence (or absence) of a primary user. Recently, FCC proposed rules in 2004 for unlicensed operations in the TV white spaces (UHF bands). So wireless devices (such as. Legacy medical telemetry equipment) are given permissions to operate in the TV bands. In these regulations, spectrum sensing plays a major role.

\section{LITERATURE SURVEY}

Detection performance of an energy detector used for cooperative spectrum sensing in a cognitive radio network is investigated in paper [10] over channels with both multipath fading and shadowing. The spectrum sensing scheme used is energy detection method.

In paper [20], a survey of all the available spectrum sensing methodologies for cognitive radio is done. Various aspects of spectrum sensing problem are studied. It also introduces the multi-dimensional spectrum sensing concept. Challenges associated with spectrum sensing are described. Reviews of the spectrum sensing methods are performed. The paper also explains the cooperative spectrum sensing concept. External sensing algorithms are studied. In addition to these, statistical modeling of network traffic and utilization of these static models for the accurate prediction of the behavior of primary user is done.

Paper [2] examines how cognitive radio can be utilized in short range systems based on Ultra-Wideband (UWB). UWB is a technology in wireless communication used for high speed data transmission with low power utilization with applications in military, radar, sensor, tracking, data collection or even commercial application. UWB can move between very low data rate or very high data rate, also between short range and long range distance applications. Impulse radio UWB shows 
some characteristics in short-range communications with varieties of throughput options that include high data rates. The strong synergy between the aims of cognitive radio and features of IR-UWB has been shown in this paper.

Paper [8] explains that the detection performance is often compromised with multipath fading, shadowing and receiver uncertainty issues. To mitigate the impact of these issues, cooperative spectrum sensing has been shown to be an effective method to improve the detection performance by exploiting spatial diversity.

Paper [11] proposes light-weight cooperation in sensing based on hard decisions to reduce the sensitivity requirements of individual radios. Cognitive Radios have been advanced as a technology for the opportunistic usage of under-utilized spectrum since they are able to sense the spectrum and use frequency bands if and only if no primary user is detected. However, the required sensitivity is very demanding since any individual radio might face a deep fade.

\section{CYCLOSTATIONARY FEATURE DETECTION}

A cyclostationary process has statistical properties that vary periodically over time. Cyclostationary feature detection method deals with the inherent cyclostationary properties or features of the signal. Such features have a periodic statistics and spectral correlation that cannot be found in any interference signal or stationary noise. It exploits this periodicity in the received primary signal to identify the presence of primary users, and that is why the cyclostationary feature detection method possesses higher noise immunity than any other spectrum sensing method. In this method, the cyclic spectral correlation function (or SCF) is the parameter that is used for detecting the primary user signals.

Cyclostationary spectrum sensing method performs better than other detection schemes such as energy detection method in low SNR regions, because of its noise rejection capability. This occurs because noise is totally random and does not exhibit any periodic form of behaviour. When we have no prior knowledge about primary user's waveform, which is the scenario in real life, then best technique to be adopted is cyclostationary feature detection. As an advantage, the cyclostationary spectrum sensing method can be used to find out the type of modulation scheme used by the primary user signal.

At the same time, the cyclostationary method has some disadvantages too. These include spectral leakage of high amplitude signals, their non-linearity etc. The method is computationally complex and hence requires significantly longer observation time and also costs high [1]. Also, when an insufficient number of samples are used, the detection performance will degrade due to the poor estimate of the cyclic spectral density.

\section{SPECTRAL CORRELATION FUNCTION}

Two-dimensional spectral correlation is the way to extract the periodic features of the primary user signal. These signals are cyclostationary processes that are periodic in time t. They also possess a periodic autocorrelation function.

$$
R_{y}(t+\tau)=R_{y}\left(t+T_{0}, \tau\right)
$$

The Fourier transform of the autocorrelation function is given as follows:

$$
R_{y}^{\alpha}(\tau)=\lim _{T \rightarrow \infty} \frac{1}{T} \int_{T} y\left(t+\frac{\tau}{2}\right) y\left(t-\frac{\tau}{2}\right) e^{-\mathrm{j} 2 \pi \alpha \mathrm{t}} d t
$$

In the above equation, $\alpha$ is the fundamental cyclic frequency and $R_{y}^{\alpha}(\tau)$ is the cyclic autocorrelation function. The spectrum correlation density function is obtained by cyclic autocorrelation function and can separate the Wide-Sense Stationary noise from the primary users signal. When the parameter $\alpha=0$ the SCF becomes power spectral density. Thus the spectral correlation function (SCF) can be defined as follows:

$$
S_{y}^{\alpha}(f)=\lim _{\Delta t \rightarrow \alpha T \rightarrow \alpha} \lim _{\Delta t} \frac{1}{\Delta t} \frac{1}{T} \int_{-\Delta t / 2}^{\Delta t / 2} Y_{T}\left(t, f+\frac{\alpha}{2}\right) Y_{T}^{*}\left(t, f-\frac{\alpha}{2}\right) d t
$$

When SCF is plotted, the occupancy status of the spectrum can be found out. If a primary user signal is present in the operating frequency range, the SCF gives a peak at its centre. The peak will not be present in the case when there is no primary user signal present in the concerned frequency range.

In addition to this, the SCF can be used to find out the type of modulation scheme used by the primary user signal. This can be achieved by counting the number of secondary peaks at the double frequencies. If the modulation scheme involved is BPSK, there will be single secondary peaks at the double of operating frequency. Instead if the modulation scheme involved is QPSK, there will be two such secondary peaks at the double of operating frequency.

\section{MOTIVATION BEHIND COOPERATION}

The main idea of cooperative spectrum sensing is to improve its performance by exploiting the spatial diversity in the observations of spatially located cognitive relay nodes. Cognitive relays share their sensed information for making a combined decision which will be more accurate than the individual nodes decisions under non cooperation situation. The performance improvement due to spatial diversity is called as the cooperative gain [8]

Cooperative spectrum sensing overcomes the hidden node problem, multi path fading and shadowing. It also improves the probability of detection. Probability of Detection (Pd) can be defined as the probability that the primary user will be detected under the given conditions if the user is in the region of the spectrum being searched.

\section{SIMULATION MODEL OF PROPOSED SYSTEM}

Some random signal is taken as the primary user signal. The signal from primary user is modulated and then relayed by multiple cognitive relays and the data is send to the fusion center. The relaying method involved here is amplify and forward relaying. At the fusion center, either hard or soft combination schemes or any of the majority combining rules, AND, OR rules etc can be used. Then after passing through the wireless channel (here AWGN is used as the channel) it reaches the cyclostationary feature detector (CFD) section. This section involves a band pass filter, an analog to digital convertor, finding the fast Fourier transform, correlating, 
averaging and feature detection. Final output of the CFD section is the estimated cyclic spectral correlation function (SCF). This spectral correlation function is analyzed to detect the signals in the cyclostationary-based spectrum sensing method. CFD output is demodulated using an appropriate scheme before it reaches the primary user's receiver.

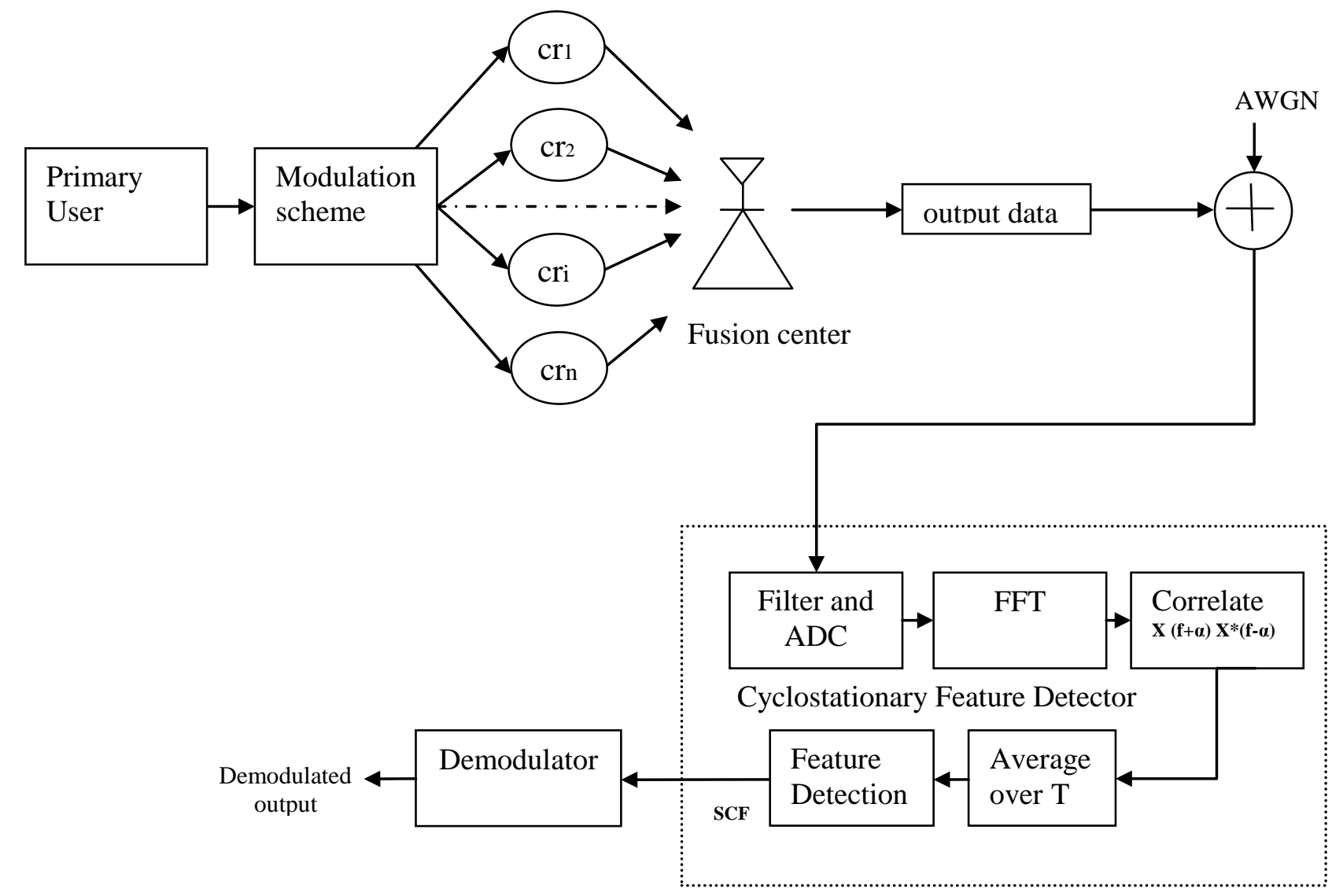

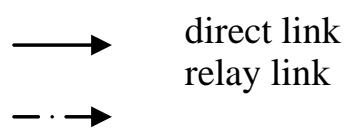

cr1,cr2,..cri...crn:

cognitive relays
Figure 1. Block diagram

\section{RESULTS \& DISCUSSIONS}

A random input is taken and modulated, by either BPSK or QPSK scheme. The operating frequency is chosen to be 1000 $\mathrm{Hz}$ here. After adding noise, it is passed through the AWGN channel. The received signal is demodulated and fed to the cyclostationary feature detector (CFD) block.

The output plot thus obtained is the cyclic SCF. The cyclic SCF contains a peak at the centre if there is a primary user in the spectrum (as in Fig. 2). In the absence of any primary user, (ie. equivalent to inputting all zeros) the cyclic SCF will not be having any peak (as in Fig. 3). Thus the outputs of feature detector with and without a primary user are obtained.

Table 1. Input Parameters

\begin{tabular}{|l|l|}
\hline Detection scheme & $\begin{array}{l}\text { Cyclostationary Feature } \\
\text { Detection }\end{array}$ \\
\hline Operating frequency & $1000 \mathrm{~Hz}$ \\
\hline
\end{tabular}

\begin{tabular}{|l|l|}
\hline Modulation scheme & BPSK, QPSK \\
\hline Channel model & AWGN \\
\hline Number of relays & 1 to 10 \\
\hline $\begin{array}{l}\text { Probability of False } \\
\text { Alarm }\end{array}$ & $0.1: 0.1: 1$ \\
\hline Fusion rule & AND \\
\hline
\end{tabular}

Table 2. Output Parameters

\begin{tabular}{|l|l|}
\hline Cyclic Spectral Correlation Function & SCF \\
\hline Probability of Detection, Pd & 0 to 1 \\
\hline
\end{tabular}




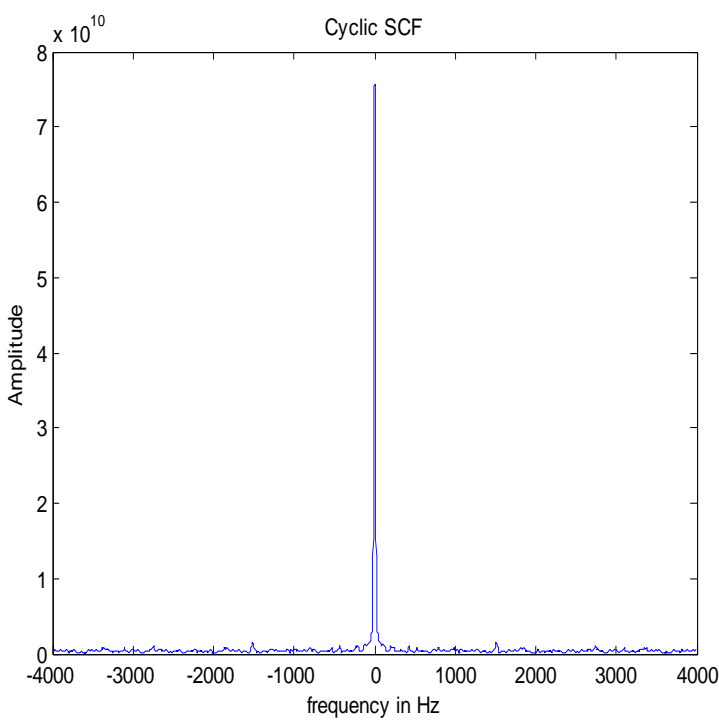

Figure 2. Cyclic SCF when Primary User is present

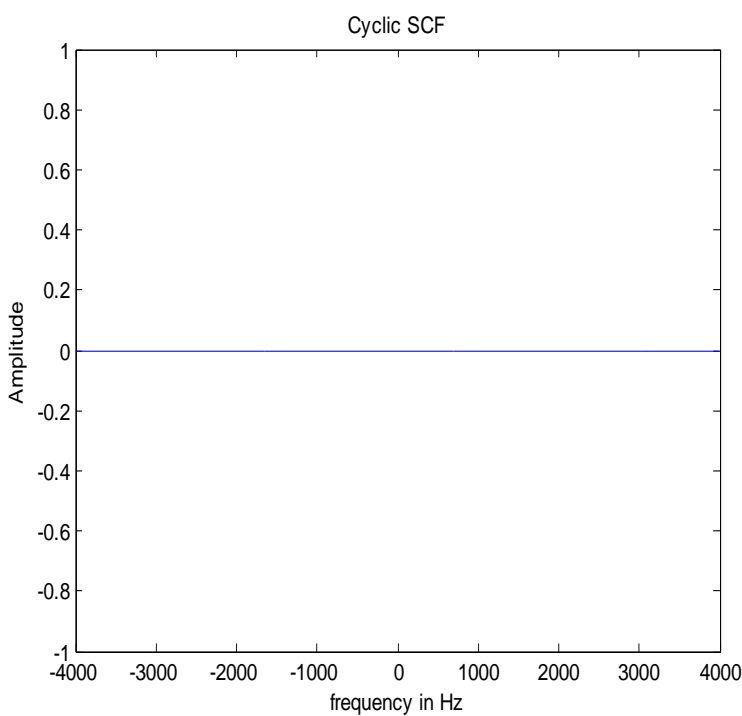

Figure 3. Cyclic SCF when Primary User is absent Here the operating frequency is taken as $1000 \mathrm{~Hz}$. The sampling frequency is taken as twenty times the operating frequency (ie. $20 \mathrm{KHz}$ ). Figure 4 shows the cyclic SCF when the input signal at the primary is modulated using BPSK scheme. At the double frequencies, ie. nearer to $2000 \mathrm{~Hz}$, single secondary peaks are visible which indicates that the modulation involved is BPSK.

Similarly in Figure 5, the cyclic SCF when the input signal at the primary is modulated using QPSK scheme is shown. At the double frequencies, ie. nearer to $2000 \mathrm{~Hz}$, two secondary peaks are visible as against single secondary peaks obtained in the case of BPSK. This indicates that the modulation technique involved is QPSK.

In Figure 6 the number of cognitive relays that cooperate in sensing is plotted as against the probability of detection. $\mathrm{P}_{\mathrm{d}}$ value for 4 users is 0.45 but $P_{d}$ value for 5 users is 0.52 . This shows that the detection probability, $P_{d}$ increases as the number of cognitive radio users, $\mathrm{n}$ increase.

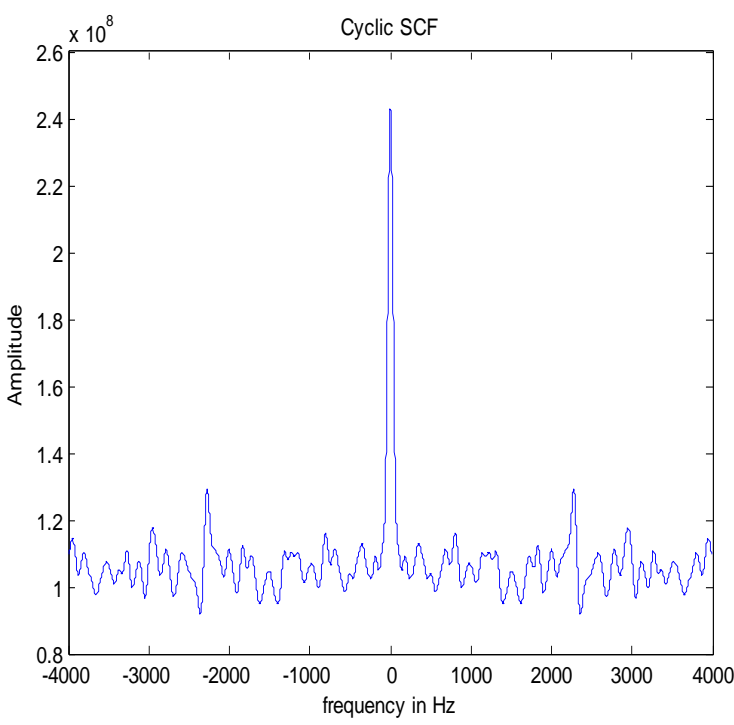

Figure 4. Cyclic SCF when Primary User signal is BPSK modulated

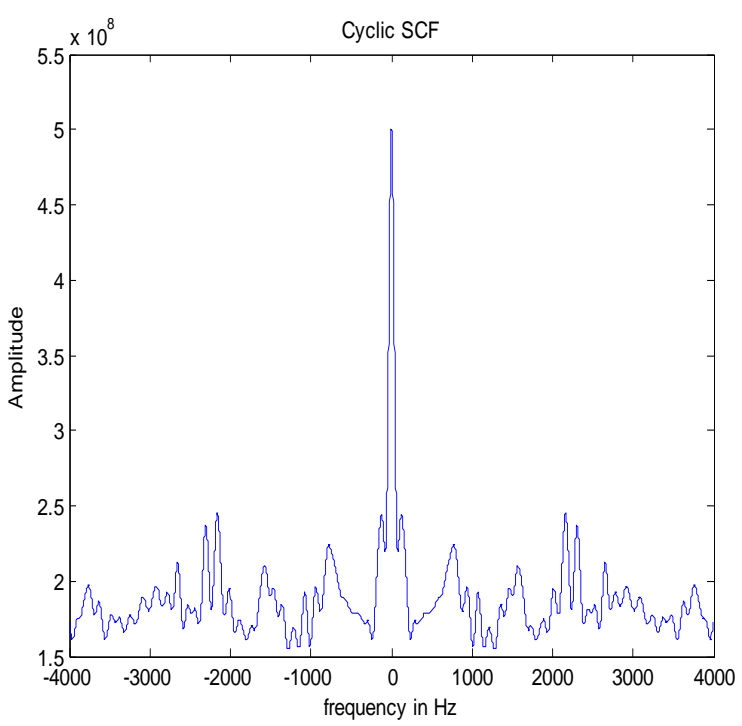

Figure 5. Cyclic SCF when Primary User signal is QPSK modulated

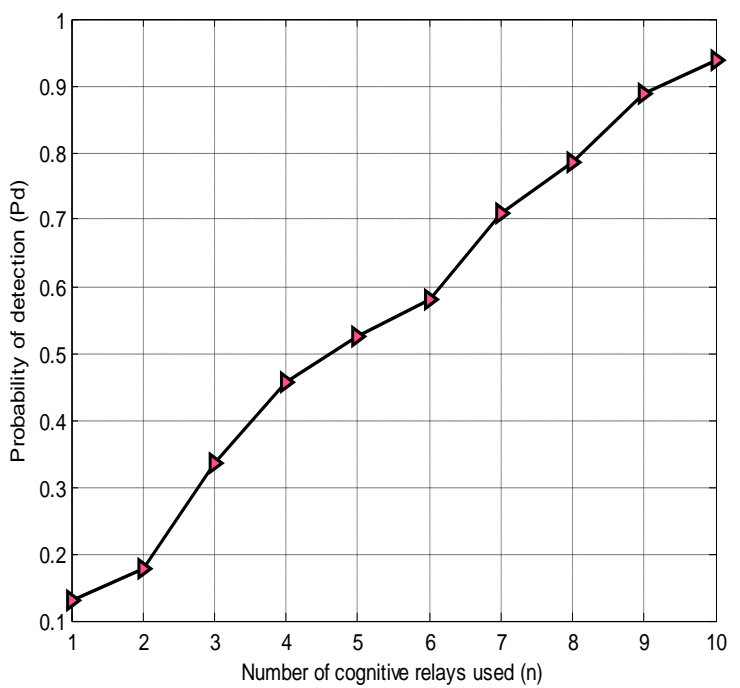

Figure 6. Plot of $\mathbf{n}$ against $\mathbf{P d}$ 


\section{CONCLUSION}

The cognitive radio spectrum sensing can be performed most reliably and efficiently by using the cyclostationary feature detection method. The cyclostationary detectors utilize the inherent periodicity of the modulated signals. Even though this method increases the complexity of the system, it is worth the risk since its noise immunity is immensely high. Also the modulation scheme employed by the primary user at the transmitter section can be accurately guessed using this method. This is a major advancement of CFD method over all other available spectrum sensing schemes. Moreover, cooperative spectrum sensing is performed which helps to overcome the multi path fading and shadowing effects that are likely to occur in any non-cooperative sensing scheme. The simulation results for cooperative spectrum sensing shows that performance improves if the detection is performed with more number of cognitive relays. Thus cyclostationary cooperative spectrum sensing is implemented.

\section{REFERENCES}

[1] A. Sahai and D. Cabric, (Nov. 2005), "Spectrum sensing: fundamental limits and practical challenges," IEEE Proceedings of International Symposium on New Frontiers in Dynamic Spectrum Access Networks (DySPAN), (Baltimore, MD).

[2] Abdullah Al-Mamun, Mohammad Rafiq Ullah,(2011), "Cognitive Radio for Short Range Systems based on Ultra-WideBand", Department of Signal Processing, Blekinge Institute of Technology.

[3] Danda B. Rawat and Gongjun Yan, (2011), "Spectrum Sensing Methods And Dynamic Spectrum Sharing In Cognitive Radio Networks: A Survey", Department Of Electrical And Computer Engineering, Old Dominion University, USA, International Journal Of Research And Reviews In Wireless Sensor Networks, vol. 1, no. 1.

[4] Danijela Cabric, Shridhar Mubaraq Mishra, Robert W.Brodersen, "Implementation Issues in Spectrum Sensing in Cognitive Radios", Berkeley Wireless Research Center, University of California, Berkeley.

[5] FCC, (Nov. 2002), "Spectrum policy task force," Tech. Rep.

[6] Gardner, W.A. (1993),"An Introduction to Cyclostationary Signals, Chapter1, Cyclostationarity in Communications and Signal Processing", IEEE Press, Piscataway, NJ.

[7] Hao Hu,(2009), "Cyclostationary Approach to signal detection and Classification in Cognitive radio systems", Beijing University of Posts and Telecommunications, P.R.China.

[8] Ian F. Akyildiz, Brandon F. Lo, Ravikumar Balakrishnan,( December 2010), "Cooperative spectrum sensing in cognitive radio networks: A survey", ELSEVIER, Physical Communication, Broadband Wireless Networking Laboratory, School of Electrical and Computer Engineering, Georgia Institute of Technology, Atlanta, GA, 30332, United States, pp. 4062.
[9] J. Ma, G.Y. Li, and B.H. Juang,( May 2009), "Signal processing in cognitive radio," Proceedings IEEE, vol. 97, no. 5, pp. 805-823.

[10] J. Mitola , III and G. Q. Maquire , Jr, (Aug. 1999)“Cognitive radio: Making software radios more personal," IEEE Personal Communications, vol. 6, no. 4, pp. 13-18, 1070-9916/99/\$10.00 @ 1999 IEEE.

[11] J. Mitola, "Software Radio: Wireless Architecture for the 21st Century", ISBN 0-9671233-0-5.

[12] J. Mitola, (Dec. 1998), “Cognitive Radio,” Licentiate proposal, KTH, Stockholm, Sweden.

[13] Jarmo Lundén, Visa Koivunen, , Anu Huttunen, and H. Vincent Poor, (November 2009), "Collaborative Cyclostationary Spectrum Sensing for Cognitive Radio Systems", IEEE Transactions On Signal Processing, vol. 57 , no. 11

[14] M. Gandetto, C. Regazzoni,( April, 2007), "Spectrum sensing: A distributed approach for cognitive terminals," IEEE Journal Selected Areas in Comm., vol. 25, pp. 546557.

[15] Mansi Subhedar and Gajanan Birajdar, (2011), "Spectrum Sensing Techniques In Cognitive Radio Networks: A Survey", International Journal Of NextGeneration Networks (Ijngn) Vol.3, No.2, Department Of Electronics and Telecommunication Engineering, Sies Graduate School Of Technology, Navi Mumbai, India.

[16] S. Haykin, (Feb. 2005) , "Cognitive radio: Brainempowered wireless communications", IEEE Journal on Selected Areas of Communication, vol. 23, no. 2, pp. 201-220.

[17] S. Haykin, D. J. Thomson, and J. H. Reed,(2009), "Spectrum sensing for cognitive radio," Proceedings of the IEEE, vol. 97, no. 5, pp. 849-877.

[18] Saman Atapattu, Chintha Tellambura and Hai Jiang, (2011), "Energy Detection Based Cooperative Spectrum Sensing In Cognitive Radio Networks" , IEEE Transactions On Wireless Communications, Vol.10, No. 4, pp.1232-1241

[19] Shridhar Mubaraq Mishra, Anant Sahai and Robert W. Brodersen, "Cooperative Sensing among Cognitive Radios", University of California, Berkeley, California.

[20] Tevfik Y"Ucek and H"Useyin Arslan, (2009), "A Survey Of Spectrum Sensing Algorithms For Cognitive Radio Applications", IEEE Communications Surveys \& Tutorials, Vol. 11, No. 1, First Quarter 2009, pp.126-130.

[21] Vinod Sharma and Arunkumar Jayaprakasam, "An Efficient Algorithm For Cooperative Spectrum Sensing In Cognitive Radio Networks", Dept Of Electrical Communication Engineering, Indian Institute Of Science, Bangalore- 560012.

[22] Waleed Ejaz, (2008), "Spectrum sensing in Cognitive Radio Networks", National University of Sciences and Technology, Pakistan. 